\title{
Airfoil trailing-edge noise prediction combining a random particle-mesh method with a Helmholtz solver
}

\author{
A.H. Kadar*, S. Le Bras ${ }^{\dagger}$, and H. Bériot ${ }^{\ddagger}$ \\ Siemens Industry Software NV, Interleuvenlaan 68, 3001 Leuven, Belgium \\ V. Korchagin ${ }^{\S}$, W. De Roeck ${ }^{\mathbb{I}}$, and W. Desmet" \\ KU Leuven, Department of Mechanical Engineering, Celestijnenlaan 300, 3001 Leuven, Belgium \\ C. Schram** \\ von Karman Institute for Fluid Dynamics, Chaussée de Waterloo 72, 1640 Rhode-St-Genèse, Belgium
}

\begin{abstract}
In this study, a computational aeroacoustic strategy is presented for the prediction of airfoil trailing-edge noise. This strategy is based on the use of a random particle-mesh method to compute noise sources, and the use of a Helmholtz solver to propagate sound waves. The random particle-mesh method is a stochastic approach that reconstructs sound sources from the steady mean flow statistics provided by an incompressible Reynolds-averaged Navier-Stokes simulation. The sound propagation is carried out in the frequency domain using a high-order finite element solver. For low-Mach number flows, assuming negligible mean flow convection effects, a non-homogeneous Helmholtz equation with a source term provided by the random particle-mesh method is solved. The performance of the numerical strategy is evaluated by simulating the sound radiated from the trailing-edge of a controlled-diffusion airfoil. The flow around the airfoil is characterized by a Mach number of 0.047 and a Reynolds number based on the airfoil chord length of $R e_{\mathrm{c}}=1.6 \times 10^{5}$. The frequency domain results are found to be in good agreement with experimental data from the literature and with a full time-domain computation, used as reference. In particular, the trailing-edge noise radiated in the far-field region is well predicted.
\end{abstract}

\section{Introduction}

$\mathrm{D}$ ESPITE advances in high-performance computing technologies, direct noise computations from the resolution of the compressible Navier-Stokes equations remain computationally very expensive for regular iterative design processes in industry [1]. This is mainly due to the high computational costs associated with the resolution of unsteady turbulent flow scales generating noise, and to the long time signals required for broadband noise predictions. Alternatively, in order to model sound sources at a reasonable cost, stochastic methods, such as the random particle-mesh (RPM) technique, have been developed in order to compute aeroacoustic sources from a steady mean-flow field.

The RPM technique, initially introduced by Ewert et al. [2-4], consists in reconstructing a stochastic turbulent field to define aeroacoustic source terms. The turbulent field is built by synthesizing velocity fluctuations that accurately represent the turbulent kinetic energy, the integral length scale and time scale distributions provided by a Reynolds-averaged Navier-Stokes (RANS) computation. The propagation of sound waves is then carried out by combining the source terms computed from RPM with acoustic perturbation equations (APE) [5]. In practice, the sound propagation is usually performed in the time domain. Such numerical approach has been successfully applied to predict trailing-edge noise [3, 6], slat noise [4], fan interaction noise [7] and HVAC duct noise [8].

An alternative technique is to perform the sound propagation in the frequency domain. Computational aeroacoustic (CAA) work-flows, based on stochastic sound source generation and sound propagation in the frequency domain, have

\footnotetext{
*PhD Student.

${ }^{\dagger}$ Research Engineer.

$\doteqdot$ Research Manager.

$\S$ PhD Student.

IIAssistant Professor.

" Full Professor.

** Professor.
} 
previously been investigated in the literature. For instance, in Cozza et al. [9], the sound sources are calculated using a digital filter-based stochastic turbulence method and the acoustic field is computed from the APE equations in the frequency domain. In Casalino et al. [10], a stochastic Fourier method is used to compute the source terms of Howe's acoustic analogy [11], which is solved in frequency domain using a finite element method (FEM).

In this study, the sound sources are stochastically computed from RPM [7] and the sound propagation is carried out in the frequency domain. For this purpose, the APE equations are reformulated in the frequency domain. For low Mach number flows, neglecting the mean-flow convection and refraction effects, the sound propagation model becomes equivalent to the resolution of a non-homogeneous Helmholtz equation, which can be efficiently solved using a high-order finite element approach [12]. The CAA workflow proposed in the present work is depicted in Fig.1. This numerical strategy is evaluated by simulating the sound generated by a low Mach number flow past the trailing-edge of a controlled-diffusion airfoil.

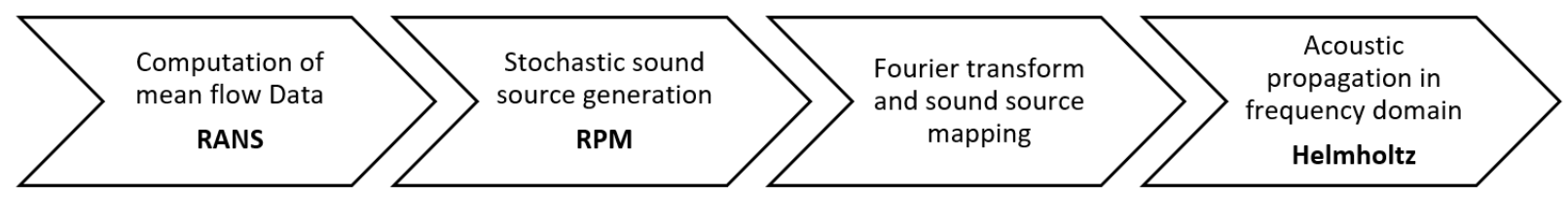

Fig. 1 Schematic representation of the CAA work-flow based on RPM.

The paper is organized as follows. In section II, the numerical strategy combining RPM with the FEM solver is described. The mapping of the sources from the RPM grid to the FEM mesh is also discussed, together with practical considerations on the choice of the time step for RPM computations. In section III, the application to CD airfoil trailing-edge noise is presented, and the results are compared with the experimental data from the literature [13].

\section{Methodology}

\section{A. Generating turbulent velocity fluctuations using the random particle-mesh method}

\section{Theoretical background}

In the framework of the RPM method, turbulent velocity fluctuations are reconstructed from a spatial filtering of a given stochastic field. A statistical description of the mean flow is required as input, including the mean flow density $\rho_{0}$, velocity $\mathbf{u}_{0}$, turbulent kinetic energy $k$, integral length scale $\Lambda$ and integral time scale $\tau$. These input data are typically provided by a preliminary RANS computation. In the following, the procedure to determine the velocity fluctuations in 2D from the RPM method is described. It is illustrated for an airfoil trailing-edge noise application in Fig. 2. First, the RPM computational domain, denoted $\Omega_{0}$, is defined in the sound source region. In Fig. 2, it corresponds to the rectangular box surrounding the airfoil trailing-edge. Assuming an incompressible flow, the velocity fluctuations can be expressed in terms of a stream function $\psi$. The function $\psi$ at point $\mathbf{x}$ is defined by the convolution product:

$$
\psi(\mathbf{x}, t)=\int_{\Omega_{0}} G\left(\mathbf{x}-\mathbf{x}^{\prime}\right) W\left(\mathbf{x}^{\prime}, t\right) \mathrm{d} \mathbf{x}^{\prime},
$$

where $G$ is a filter kernel in space, and $W$ is a convected stochastic field. The filter $G$ determines the turbulence spatial properties, and is derived from an energy spectrum model. In this work, a Gaussian energy spectrum is used, with the assumption of locally homogeneous and isotropic turbulence [7]:

$$
G\left(\mathbf{x}-\mathbf{x}^{\prime}\right)=\sqrt{\frac{2 \rho_{0} k}{\pi}} \exp \left(\frac{-\pi\left|\mathbf{x}-\mathbf{x}^{\prime}\right|^{2}}{2 \Lambda^{2}}\right),
$$

where the mean flow quantities $\rho_{0}, k$, and $\Lambda$ are evaluated at $\mathbf{x}^{\prime}$. The convected stochastic field $W$ in Eq. (1) verifies the following properties:

$$
\begin{aligned}
\left\langle W\left(\mathbf{x}^{\prime}, t\right)\right\rangle & =0, \\
\left\langle W\left(\mathbf{x}^{\prime}, t\right) W\left(\mathbf{x}^{\prime}+\mathbf{r}, t+s\right)\right\rangle & =\rho_{0}(\mathbf{x})^{-1} \delta\left(\mathbf{r}-\mathbf{u}_{0} s\right) \exp (-s / \tau),
\end{aligned}
$$


where the operator $\langle\ldots\rangle$ denotes an ensemble average and $\delta$ is the Dirac delta function. In Eq. (4), the exponential term prescribes evolving turbulence with exponential time-decorrelation. The exponential time-decorrelation is realized using the Langevin equation [14] given by

$$
\frac{D_{0}}{D t} W\left(\mathbf{x}^{\prime}, t\right)=\frac{-1}{\tau} W\left(\mathbf{x}^{\prime}, t\right)+\sqrt{\frac{2}{\tau}} \varsigma\left(\mathbf{x}^{\prime}, t\right)
$$

where $D_{0} / D t=\partial / \partial t+\mathbf{u}_{0} \cdot \nabla$ is the total derivative and $\varsigma$ is a zero-mean white-noise field with variance $\rho_{0}(\mathbf{x})^{-1}$. Finally, the velocity fluctuations $\mathbf{u}^{\prime}$ are computed from the curl of the stream function $\psi$ as $\mathbf{u}^{\prime}=\nabla \times \psi \hat{\mathbf{k}}$, where $\hat{\mathbf{k}}$ is the unit vector in z-direction. It yields:

$$
u_{i}^{\prime}(\mathbf{x}, t)=\int_{\Omega_{0}}\left(\epsilon_{i j} \frac{\partial G\left(\mathbf{x}-\mathbf{x}^{\prime}\right)}{\partial x_{j}}\right) W\left(\mathbf{x}^{\prime}, t\right) \mathrm{d} \mathbf{x}^{\prime},
$$

where $\epsilon_{i j}$ is the Levi-Civita symbol.

\section{Numerical implementation}

A fully Lagrangian approach is followed for the RPM implementation, as proposed in [7]. In this method, the stochastic field is represented by particles carrying values which are initially randomly distributed in the fluid region and then convected by the RANS mean flow velocity. Particles are seeded at the inflow boundary and they leave the domain at the outflow boundary (see Fig. 2). The fluctuations are computed on a uniform Cartesian grid of size $h_{\text {RPM }}$ using a Lagrangian formulation of Eq. (6):

$$
u_{i}{ }^{\prime}(\mathbf{x}, t)=\sum_{n=1}^{N}\left(\epsilon_{i j} \frac{\partial G\left(\mathbf{x}-\mathbf{x}_{\mathbf{n}}^{\prime}\right)}{\partial x_{j}}\right) W_{n}(t),
$$

where $N$ denotes the total number of particles and $W_{n}$ is the weighted average of $W$ over a fluid element surrounding the $n^{\text {th }}$ particle [7]. The value of $W_{n}$ is updated at each time step using the Langevin equation (5), which is numerically integrated using a 4th order Runge-Kutta (RK) time integration scheme. In practice, we restrict the number of particles used in the filtering process in Eq. (7), and the domain of influence of each particle is set to a circle of radius $2 \Lambda$ [15].

The cost of the RPM method is mainly driven by the filtering operation in Eq. (7), and it scales linearly with the number of velocity update operations. In order to reduce the computational effort, the time step $\Delta t_{\mathrm{ac}}$ at which the velocity fluctuations (7) and thus the acoustic sources are calculated may be chosen as a multiple of the time step $\Delta t_{\mathrm{RPM}}$ at which the value of $W_{n}$ and the particle position are updated. The determination of the time steps $\Delta t_{\mathrm{RPM}}$ and $\Delta t_{\mathrm{ac}}$ is discussed in more detail in section II.D.

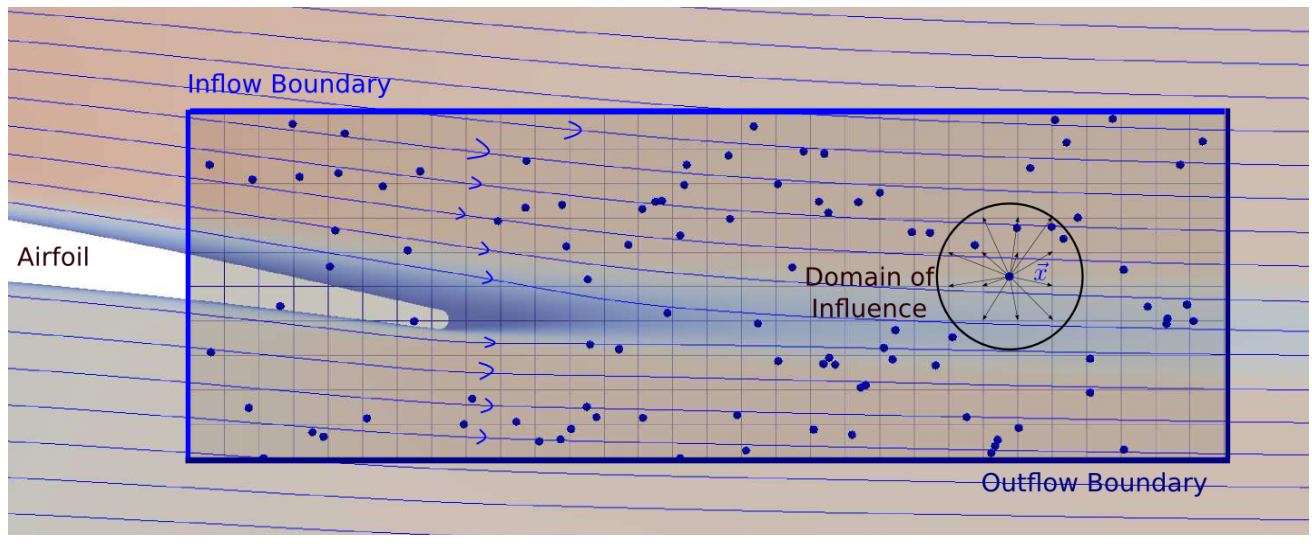

Fig. 2 Sketch of the 2D computational domain used for the RPM method.

\section{B. Propagation model in the time domain}

In this study, the time domain propagation model relies on the acoustic perturbation equations (APE-4) proposed in [5]. The APE-4 formulation constitutes an aero-acoustic analogy. The left-hand side describes linear acoustic 
perturbations in the presence of a non-uniform mean flow. The right-hand side includes a vortex sound source, involving the velocity fluctuations generated using the RPM method and the mean velocity and vorticity obtained from RANS. The APE-4 equations, considering only the vortex sound source term on the right-hand side of the momentum equation, are defined as follows:

$$
\left\{\begin{array}{l}
\frac{\partial p^{\prime}}{\partial t}+c_{0}^{2} \nabla \cdot\left(\rho_{0} \mathbf{u}^{\prime}+\frac{p^{\prime}}{c_{0}^{2}} \mathbf{u}_{0}\right)=0, \\
\frac{\partial \mathbf{u}^{\prime}}{\partial t}+\nabla\left(\mathbf{u}_{0} \cdot \mathbf{u}^{\prime}\right)+\nabla\left(\frac{p^{\prime}}{\rho_{0}}\right)=-\mathbf{L}^{\prime}
\end{array}\right.
$$

where the quantities with prime .' are the unknown acoustic variables (pressure $p^{\prime}$ and velocity $\mathbf{u}^{\prime}$ ), and $c_{0}$ is the speed of sound. The Lamb vector $\mathbf{L}^{\prime}$ corresponding to the source term in Eq. (9) is defined as

$$
\mathbf{L}^{\prime}=\omega_{0} \times \mathbf{u}^{\prime}+\omega^{\prime} \times \mathbf{u}_{0}+\omega^{\prime} \times \mathbf{u}^{\prime}-\left\langle\omega^{\prime} \times \mathbf{u}^{\prime}\right\rangle
$$

The non-linear terms in Eq. (10) can be considered smaller than the linear terms [4], and can thus be discarded. It results in the following approximation:

$$
\mathbf{L}^{\prime} \approx \omega_{0} \times \mathbf{u}^{\prime}+\omega^{\prime} \times \mathbf{u}_{0}
$$

For low Mach number applications, the non-uniform mean flow $\mathbf{u}_{0}$ can be neglected from the acoustic propagation operator. Further assuming uniform mean density $\rho_{0}$, the APE-4 equations (8) and (9) reduce to the following set of equations:

$$
\left\{\begin{array}{l}
\frac{\partial p^{\prime}}{\partial t}+c_{0}^{2} \rho_{0} \nabla \cdot \mathbf{u}^{\prime}=0 \\
\frac{\partial \mathbf{u}^{\prime}}{\partial t}+\frac{1}{\rho_{0}} \nabla p^{\prime}=-\mathbf{L}^{\prime}
\end{array}\right.
$$

In a recent work [15], a numerical strategy was used to approximate the solution to the propagation equations (12) and (13) by using a high-order quadrature-free Discontinuous Galerkin (DG) method [16]. The same approach is applied here, to serve as a reference for the verification of the frequency domain propagation model, which is introduced hereafter.

\section{Propagation model in the frequency domain}

The previous system of equations is first reformulated into a classical scalar wave equation, by combining the time derivative of Eq. (12) and the divergence of Eq. (13):

$$
\frac{\partial^{2} p^{\prime}}{\partial t^{2}}-c_{0}{ }^{2} \nabla^{2} p^{\prime}=c_{0}^{2} \rho_{0} \nabla \cdot \mathbf{L}^{\prime} .
$$

The fluctuating pressure $p^{\prime}$ and the Lamb vector $\mathbf{L}^{\prime}$ are then written as inverse Fourier transforms:

$$
\begin{aligned}
p^{\prime}(\mathbf{x}, t) & =\frac{1}{2 \pi} \int_{-\infty}^{\infty} \hat{p}(\mathbf{x}, \omega) e^{-i \omega t} d \omega \\
\mathbf{L}^{\prime}(\mathbf{x}, t) & =\frac{1}{2 \pi} \int_{-\infty}^{\infty} \widehat{\mathbf{L}}(\mathbf{x}, \omega) e^{-i \omega t} d \omega
\end{aligned}
$$

where $\hat{p}$ and $\widehat{\mathbf{L}}$ are the respective Fourier coefficients and $\omega$ is the angular frequency. Substituting them in Eq. (14), results in the following non-homogeneous Helmholtz equation:

$$
\nabla^{2} \hat{p}+k_{\omega}^{2} \hat{p}=-\rho_{0} \nabla \cdot \widehat{\mathbf{L}}
$$

where $k_{\omega}=\omega / c_{0}$ is the wavenumber. It is worth noting that the Lamb vector field acts as an equivalent dipolar source term in the proposed Helmholtz analogy.

A sketch of the acoustic propagation problem applied to an airfoil is shown in Fig. 3. The resulting bounded Helmholtz problem reads:

$$
\left\{\begin{array}{l}
\nabla^{2} \hat{p}+k_{\omega}^{2} \hat{p}=-\rho_{0} \nabla \cdot \widehat{\mathbf{L}} \quad \forall x \in \Omega \\
\nabla \hat{p} \cdot \mathbf{n}=0 \quad \forall x \in \partial \Omega_{s}
\end{array}\right.
$$


where $\Omega$ is the acoustic propagation domain, $\mathbf{n}$ is the outwarding surface normal, and $\partial \Omega_{s}$ is the airfoil scattering surface on which impervious rigid wall conditions are prescribed. In addition, to represent sound radiation to the far-field, non-reflecting boundary conditions are also prescribed on the exterior surface $\partial \Omega_{\infty}$, to remove spurious reflections.

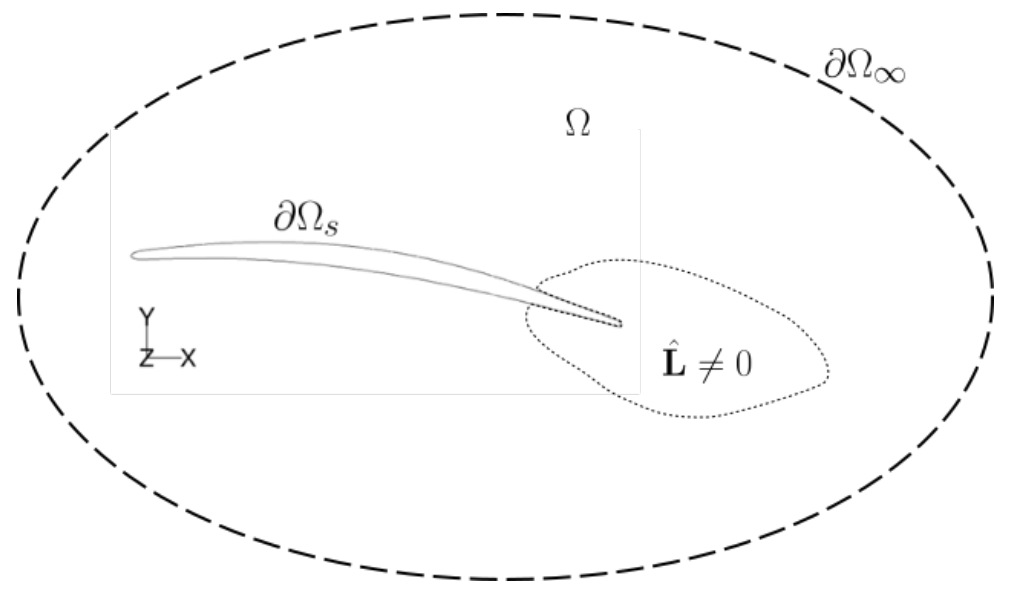

Fig. 3 Sketch of the computational domain used for the acoustic propagation.

The solution to the Helmholtz problem (18) is approximated using a classical Galerkin finite element approach. The corresponding variational formulation is given by

$$
-\int_{\Omega} \nabla q \cdot \nabla \hat{p} \mathrm{~d} \mathbf{x}+k_{\omega}^{2} \int_{\Omega} q \hat{p} \mathrm{~d} \mathbf{x}+\int_{\partial \Omega_{\infty}} q(\nabla \hat{p} \cdot \mathbf{n}) \mathrm{d} \mathbf{x}=-\int_{\Omega} q\left(\rho_{0} \nabla \cdot \widehat{\mathbf{L}}\right) \mathrm{d} \mathbf{x},
$$

where $q$ denotes the test function. In order to avoid an unnecessary numerical differentiation of the Lamb vector $\widehat{\mathbf{L}}$, which is prone to numerical errors, the right-hand-side of Eq. (20) is integrated by parts as follows:

$$
-\int_{\Omega} q\left(\rho_{0} \nabla \cdot \widehat{\mathbf{L}}\right) \mathrm{d} \mathbf{x}=\int_{\partial \Omega}-\rho_{0}(\widehat{\mathbf{L}} \cdot \mathbf{n}) q \mathrm{~d} \mathbf{x}-\int_{\Omega}-\rho_{0} \nabla q \cdot \widehat{\mathbf{L}} \mathrm{d} \mathbf{x}
$$

Considering that the Lamb vector vanishes on the scattering surface $\partial \Omega_{s}$ and the exterior surface $\partial \Omega_{\infty}$, Eq. (20) simplifies to:

$$
-\int_{\Omega} \nabla q \cdot \nabla \hat{p} \mathrm{~d} \mathbf{x}+k_{\omega}^{2} \int_{\Omega} q \hat{p} \mathrm{~d} \mathbf{x}+\int_{\partial \Omega_{\infty}} q(\nabla \hat{p} \cdot \mathbf{n}) \mathrm{d} \mathbf{x}=\int_{\Omega} \rho_{0} \widehat{\mathbf{L}} \cdot \nabla q \mathrm{~d} \mathbf{x} .
$$

In practice, the Lamb vector $\mathbf{L}^{\prime}(\mathbf{x}, t)$ is first calculated in the time domain using the RPM method described in section II.A. The coefficients $\widehat{\mathbf{L}}(\mathbf{x}, \omega)$ are then retrieved using a Fourier transform of $\mathbf{L}^{\prime}(\mathbf{x}, t)$. Finally, a locally-conformal realization of the Perfectly Matched Layers (PML) [17] is implemented around the boundary of the domain, in order to efficiently absorb outgoing waves. In practice, the PML region is automatically extruded from the convex surface $\partial \Omega_{\infty}$ using a user-defined number of layers $N_{\text {PML }}$.

The variational formulation (22) is discretized using a high-order hierarchical finite element method [18]. This approach has shown to provide drastic reductions in both memory and CPU time when compared to classical finite element models for Helmholtz problems [12]. The domain $\Omega$ is first approximated by a collection of non-overlapping quadratic 6-noded triangular elements in the physical region and 9-noded quadrangular elements in the PML region. On each element, the numerical solution is made up of high-order $H^{1}$-conforming polynomial shape functions. In this work, for the sake of simplicity, the interpolation order $p_{\mathrm{FEM}}$, is kept constant across the entire mesh. In practice, it is chosen based on the following formula:

$$
p_{\mathrm{FEM}}>\frac{k_{\omega} h_{\mathrm{max}}}{2 \pi} d_{\lambda}
$$

where $h_{\max }$ denotes the largest element size. This approach ensures that a minimum resolution rate $d_{\lambda}$ is obtained across the entire acoustic mesh, at a given frequency of interest. 


\section{Choice of the time step to update the acoustic source}

\section{Propagation in the time domain}

In the time domain propagation model presented in section II.B, the acoustic sources calculated from RPM are provided to the propagation solver at every time step $\Delta t_{\mathrm{ac}}$. In the present study, the equations (12) and (13) are solved using a 8-stage 4th order RK time integration explicit scheme [19]. For time explicit schemes, in absence of mean flow, the time step $\Delta t_{\mathrm{ac}}$ must verify the Courant-Friedrichs-Lewy (CFL) type condition

$$
\Delta t_{\mathrm{ac}} \leq v^{*} \frac{h}{c_{0} p_{\mathrm{DG}}},
$$

where $h$ is the size of the local mesh element, $p_{\text {DG }}$ is the spatial polynomial order and $v^{*}$ denotes the maximum CFL number to guarantee the scheme stability. When very fine acoustic meshes are used to represent the geometry, the value of $\Delta t_{\mathrm{ac}}$ is thus severely restricted by the size of the smallest element in the domain. For the RPM computation, the time step $\Delta t_{\mathrm{RPM}}$ can be determined following recommendations of the literature. In particular, the time step should verify $\Delta t_{\mathrm{RPM}}<\tau_{\mathrm{avg}}$, where $\tau_{\mathrm{avg}}$ is the value of the RANS turbulence time-scale averaged over the RPM computational domain [8]. In addition, the value of $\Delta t_{\mathrm{RPM}}$ should also be chosen such that the particles traverse a maximum of one computational cell between each consecutive source update. For the low Mach number applications considered in this study, the two previous criteria are generally less restrictive than the CFL condition (24), yielding $\Delta t_{\mathrm{RPM}}=\Delta t_{\mathrm{ac}}$.

In the literature, source interpolation techniques [8] have been developed in order to reduce the computational cost associated to RPM. It consists in computing the sound sources from RPM every $m \Delta t_{\mathrm{ac}}$ time steps and interpolating the value of the sources at the intermediate time steps. Such interpolation techniques are not used in the present work.

\section{Propagation in the frequency domain}

In the frequency domain propagation model, the CFL restriction (24) does not apply. Therefore, the value of the acoustic time step $\Delta t_{\mathrm{ac}}$ may be chosen higher than the value of $\Delta t_{\mathrm{ac}}$ used in the time domain propagation solver. The RPM time step $\Delta t_{\mathrm{RPM}}$ verifies the condition $\Delta t_{\mathrm{RPM}}<\tau_{\text {avg }}$ and the constraint on the particle convection speed (see section II.D.1). In this study, for the sake of simplicity, the value of $\Delta t_{\mathrm{RPM}}$ is equal to the value used in the time domain approach.

\section{E. Transferring the sources from a RPM grid to an acoustic mesh}

As described in section II.A.2, the RPM sound sources are generated on a refined uniform Cartesian grid of size $h_{\mathrm{RPM}}$. However, in practice, this initial description is most often not retained for the acoustic propagation part. In this study, the equivalent sources are transferred onto triangular unstructured acoustic meshes, on which they are interpolated, typically using high-order polynomials. Unless otherwise stated cubic Lagrange shape functions $\left(p_{\text {source }}=3\right)$ are chosen. To be more specific, the Lamb vector Fourier coefficients $\widehat{\mathbf{L}}(\mathbf{x}, \omega)$ in the $p$-FEM variational formulation (22) are interpolated using a classical 10-noded cubic triangle element. For the sake of consistency, the same approach is chosen for the source representation in the time domain DG code.

The nodal values representing the sources on the acoustic mesh are obtained through a mapping procedure. Different mapping techniques have been proposed in the literature. For instance, in the work of Ewert et al. [2], a high-order Hermite interpolation scheme is used. In the work of Martínez-Lera [20], a conservative mapping scheme is introduced. In the current study, three interpolation techniques are examined, namely an inverse distance weighting, a bilinear and a spline interpolation scheme. A brief description of these approaches is presented below and their performance in terms of accuracy is reported in Appendix.B.

1) Inverse distance weighting: The source term $\widehat{\mathbf{L}}$ is interpolated at a nodal point $\mathbf{x}_{i}$ by taking a weighted sum of the sources at the neighbouring scattered set of RPM nodes. A modified Shepard interpolation method is chosen here [20]. It is characterized by two parameters, namely the weighting function $f$ and a mapping radius $R_{\mathrm{ac}}$. The source at $\mathbf{x}_{i}$ is computed as follows:

$$
\widehat{\mathbf{L}}\left(\mathbf{x}_{i}, \omega\right)=\frac{\sum_{j} f\left(r_{i j}\right) \widehat{\mathbf{L}}\left(\mathbf{x}_{j}, \omega\right)}{\sum_{j} f\left(r_{i j}\right)}
$$

where $r_{i j}$ denotes the distance between acoustic node $i$ and surrounding RPM node $j$, and $f\left(r_{i j}\right)=\frac{R_{\mathrm{ac}}-r_{i j}}{R_{\mathrm{ac}}+4 r_{i j}}$. The support of the mapping scheme is restricted to a circle (sphere in 3D) of radius $R_{\mathrm{ac}}$ around the acoustic node $\mathbf{x}_{i}$. 
2) Bilinear interpolation: In this approach, a bilinear interpolation is carried out to interpolate the value of $\widehat{\mathbf{L}}$ at an acoustic node from the values of $\widehat{\mathbf{L}}$ at adjacent RPM nodes surrounding the interpolation point. It requires two grid points in each dimension and ensures $C^{0}$ continuity.

3) Spline interpolation: The interpolated value of $\widehat{\mathbf{L}}$ at an acoustic node is based on a cubic interpolation of the values of $\widehat{\mathbf{L}}$ at neighbouring RPM nodes. The interpolation is based on a cubic spline using not-a-knot end conditions. It requires four points in each dimension and ensures $C^{2}$ continuity. The spline interpolation has a larger memory footprint and is more computationally demanding than the linear interpolation.

\section{Application}

\section{A. Controlled-diffusion airfoil case description}

The methodology described in section II is applied to predict the trailing-edge noise radiated from a controlled diffusion (CD) airfoil investigated both experimentally [13,21] and numerically [22-24] in the literature. A 2D representation of the CD airfoil is shown in Fig. 4. The airfoil has a constant chord length of $c=0.1356 \mathrm{~m}$ and a span length of $L=0.3 \mathrm{~m}$. It has an angle of attack $\alpha=8^{\circ}$. The flow around the airfoil is characterized by a Mach number of $M=0.047$ and a Reynolds number based on the chord length of $R e_{\mathrm{c}}=1.6 \times 10^{5}$. The ambient density and sound speed are $\rho_{0}=1.225 \mathrm{~kg} / \mathrm{m}^{3}$ and $c_{0}=340 \mathrm{~m} / \mathrm{s}$.

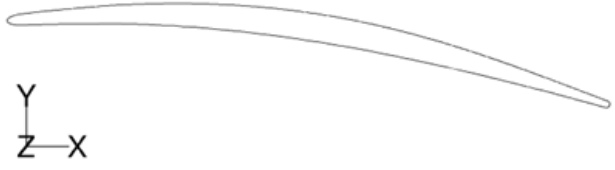

Fig. 4 Sketch of the CD airfoil in 2D.

\section{B. Sound source computation}

\section{RANS}

In order to evaluate the statistical flow properties required for the RPM method (see section II.A), an incompressible 2D RANS computation is performed. The simulation is carried out with the Finite Volume solver OpenFOAM 3.0 [25] using the SST $k-\omega$ turbulence model. The turbulence integral length scale and integral time scale are computed from the turbulent kinetic energy and specific dissipation rate as described in Appendix.A. A full description of the RANS simulation set-up and results is presented in a previous work of Kadar et al. [15].

\section{2. $R P M$}

The RPM solver [26, 27] developed at KU Leuven is used in this study. A rectangular RPM computational domain is defined around the airfoil trailing-edge. It extends from $-0.15 c$ to $0.45 c$ in the $\mathrm{x}$-direction and from $-0.08 c$ to $0.12 c$ in the y-direction. The trailing-edge is centered at $(0,0)$. The RPM domain is discretized using a uniform Cartesian grid of size $h_{\mathrm{RPM}}=0.001 \mathrm{c}$. The RANS data are linearly interpolated from the RANS mesh to the RPM grid. The number of particles $N=120,000$ is chosen considering 5 particles per minimum length scale of interest [28]. In order to assess the quality of the synthetic turbulence generated from RPM, the statistics of the reconstructed fluctuations are successfully compared with the input RANS statistics in [15].

\section{Acoustic propagation}

Once the RPM noise sources are computed, the propagation of sound waves is carried out in the frequency domain. For comparison, in the present work, sound propagation is also performed in the time domain using a DG solver [16]. The numerical set-ups for the frequency domain and the time domain approaches are presented in the following. In the two computations, the frequency range of interest for noise predictions extends from $300 \mathrm{~Hz}$ to $3 \mathrm{kHz}$, as in the experiment [13]. 


\section{Propagation in the time domain}

For the DG simulation, a 2D circular computational domain of radius $7.5 c$ centered on the airfoil trailing-edge is defined, as shown in Fig. 5(a). In order to avoid spurious reflections on the external boundary, non-reflecting boundary conditions based on characteristic flux decomposition [29] are imposed. In addition, a sponge layer [30] is added for $5 c \leq r \leq 7.5 c$ in order to damp the outgoing waves before they reach the boundary. The mesh, composed of triangular elements, is presented close to the trailing-edge in Fig. 5(b). In order to accurately represent the curved wall boundary, the geometry is discretized using quadratic elements. The smallest element size $h_{\min }=0.01 c$ is chosen in the sound source region close to the trailing-edge. The maximum element size of $h_{\max }=0.25 c$ is chosen in the damping layer. The mesh contains 37892 linear elements in the interior of the domain and 200 quadratic elements adjacent to the airfoil. The computation is performed using the polynomial order $p_{\mathrm{DG}}=3$. The time step of the acoustic simulation is fixed to $\Delta t_{\mathrm{ac}}=1.6 \times 10^{-3} \mathrm{c} / c_{0}$, in order to respect the CFL condition (24) with $v^{*}=1$.

For the definition of the acoustic sources, a RPM time signal of length $T_{\text {total }}=120 \mathrm{c} / c_{0}$ is computed. The RPM time step is equal to $\Delta t_{\mathrm{RPM}}=\Delta t_{\mathrm{ac}}=1.6 \times 10^{-3} \mathrm{c} / c_{0}$. The inverse distance weighting approach is used to interpolate the acoustic sources from the RPM grid to the DG mesh.

For the computation of the acoustic spectra in the far-field region, the fluctuating pressure $p^{\prime}$ and velocity $\mathbf{u}^{\prime}$ are recorded on a circle of radius $1.5 c$ centered at $(x=-0.5 c, y=0)$ during the period $T_{\text {total. }}$. The time signal is then divided into nine segments of length $T=24 c / c_{0}$ which overlap by $50 \%$. A Fourier transform is applied to each segment and the pressure $\hat{p_{s}}$ is extrapolated in the far-field using a 2D Kirchhoff method [31]. The nine pressure spectra thus obtained are then averaged in order to compute the average acoustic spectra $S_{\mathrm{pp}}^{\text {avg }}$ :

$$
S_{\mathrm{pp}}^{\mathrm{avg}}=\frac{1}{9} \sum_{s=1}^{9} \frac{\left|\hat{p}_{s}\right|^{2}}{T}
$$

(a)

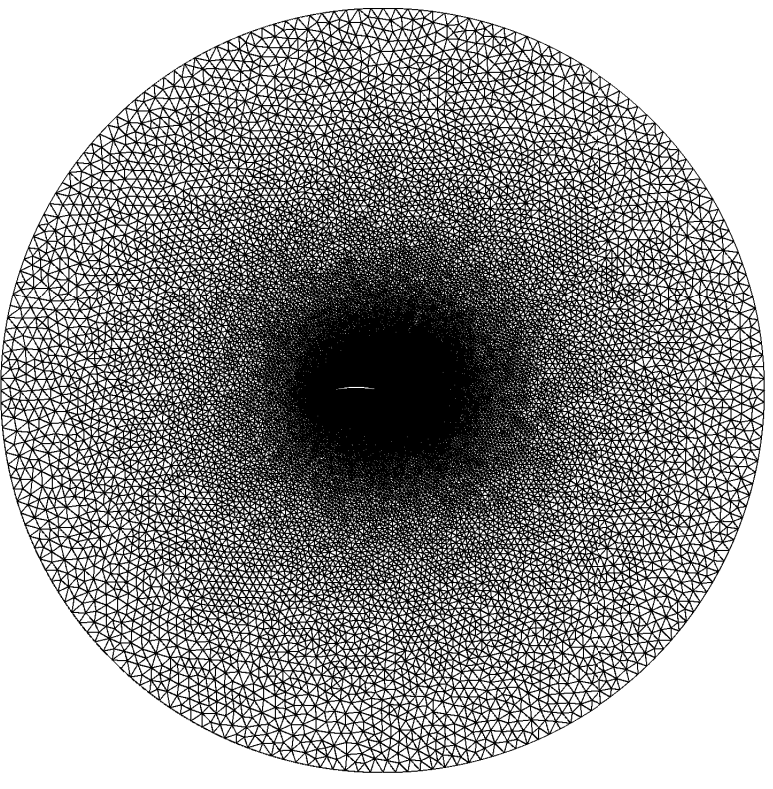

(b)

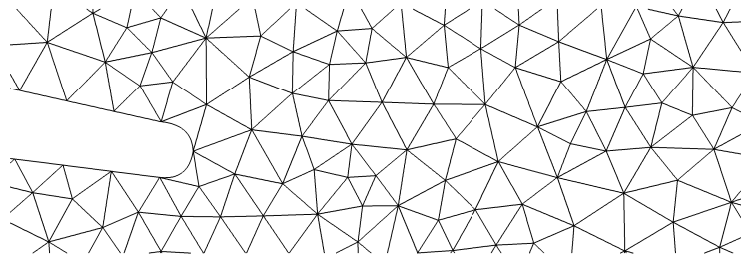

Fig. 5 Sound propagation in the time domain: a) DG computational domain and mesh, b) mesh at the airfoil trailing-edge.

\section{Propagation in the frequency domain}

As presented in section II.C, the Helmholtz problem (18) and (19) is solved using a high-order finite element (FE) approach. In this context, a 2D elliptic computational domain centered on the airfoil trailing-edge is chosen, as shown in Fig. 6(a). The ellipse is defined by a semi-major axis of length $3 c$ and a semi-minor axis of length $1.5 c$. A PML domain, 
composed of $N_{\mathrm{PML}}=5$ layers, is extruded around the physical domain to absorb all outgoing waves and avoid spurious reflections. In order to ensure a consistent geometric and source representation between both computations, the FEM and DG mesh characteristics are aligned in the near-field region, with $h_{\min }=0.01 c$, see Fig. 6(b). However, a much smaller domain size is chosen for the FEM mesh, owing to the performance of the frequency domain PML [17]. The latter does not require to be placed at large distances away from the source, thus reducing the global computational footprint of the method. The maximum element size is located in the PML and is equal to $h_{\max }=0.2 c$. The FEM mesh contains 11872 elements. The Fourier components of the Lamb vector are interpolated on the FEM acoustic mesh using the same mapping strategy as for the DG workflow. As for the fixed interpolation order $p_{\text {FEM }}$, it is adjusted at each frequency, such that a minimum resolution rate of $d_{\lambda}=7$ is obtained, as defined in Eq. (23).

For the calculation of the sound sources, a RPM time signal is generated over a period of $T_{\text {total }}=120 \mathrm{c} / c_{0}$, as in the DG computation. The RPM time step is set to $\Delta t_{\mathrm{RPM}}=1.6 \times 10^{-3} c / c_{0}$, as in DG. For the sound source update, the acoustic time step verifies the Nyquist criterion $\Delta t_{\mathrm{ac}}<0.4 c / c_{0}$, and is equal to $\Delta t_{\mathrm{ac}}=4.8 \times 10^{-2} c / c_{0}$. It is of the same order of magnitude as in $[22,24]$.

In the FEM simulation, the RPM time signal is divided into nine segments of length $T=24 c / c_{0}$ overlapping by $50 \%$. For each segment, a Fourier transform is applied to the time signal, and a sound propagation computation is carried out. The pressure and its normal derivative are recorded for each segment at the PML interface. In order to calculate the pressure spectra in the far-field region, a 2D Kirchhoff extrapolation [31] is then applied at the PML interface. The nine spectra thus obtained are then averaged in order to compute the average acoustic spectra $S_{\mathrm{pp}}^{\mathrm{avg}}$ using Eq. (26).

(a)

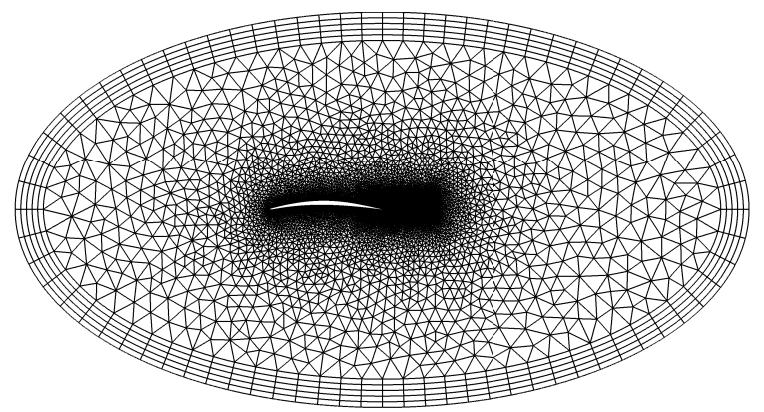

(b)

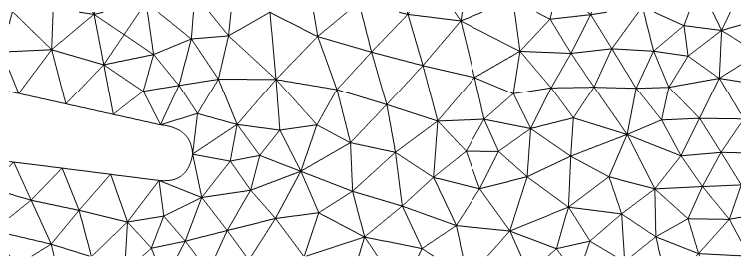

Fig. 6 Sound propagation in the frequency domain: a) FE computational domain and mesh, b) mesh at the airfoil trailing-edge.

\section{Acoustic results}

The 3D sound pressure level (SPL) at location $\mathbf{x}$ in the far-field region is estimated from the 2D spectra $S_{\mathrm{pp}}^{\text {avg }}$ using the following relation:

$$
\operatorname{SPL}(\mathbf{x}, \omega)=10 \log _{10}\left(\frac{S_{\mathrm{pp}}^{\mathrm{avg}}(\mathbf{x}, \omega) \Delta f^{\mathrm{exp}}}{p_{\mathrm{ref}}^{2}}\right)+10 \log _{10}\left(\frac{1.4}{2 \pi} \frac{L}{R} M\right)
$$

where $p_{\text {ref }}=2 \times 10^{-5} \mathrm{~Pa}$ is the reference pressure, $R$ denotes the distance from the trailing-edge to an observer. As in the experiment [13], the data are integrated into bins of width $\Delta f^{\exp }=8 \mathrm{~Hz}$. A $2 \mathrm{D}$ to 3D correction [3], corresponding to the second term in Eq. (27), is added to take into account the finite span of the airfoil.

The far-field SPL at $R=2 \mathrm{~m}$ and $\theta=90^{\circ}$ with respect to the chord line are compared with the experimental data [13] in Fig. 7. The SPL computed using the time domain and the frequency domain approaches are found to be in good agreement over the entire frequency range. The differences observed are of the order of 1 or 2 decibels, which is deemed satisfactory. As for the comparison with the experimental data, the present numerical results over-predict the experimental sound levels by 2 to $5 \mathrm{~dB}$ over the frequency range extending from $300 \mathrm{~Hz}$ to $1 \mathrm{kHz}$.

The pressure directivities are now compared for the two computations in Fig. 8. Four frequencies are examined, namely $417 \mathrm{~Hz}, 835 \mathrm{~Hz}, 1253 \mathrm{~Hz}$ and $1880 \mathrm{~Hz}$, corresponding to Helmholtz numbers $k_{c}=\omega c / c_{0}$ of $1.04,2.09,3.14$ and 4.71 respectively. The number of lobes in the directivity plots increases with the Helmholtz number, as expected [22, 24]. 
Significant discrepancies are observed between the two schemes at the highest frequency $1880 \mathrm{~Hz}$, the origin of which is under investigation.

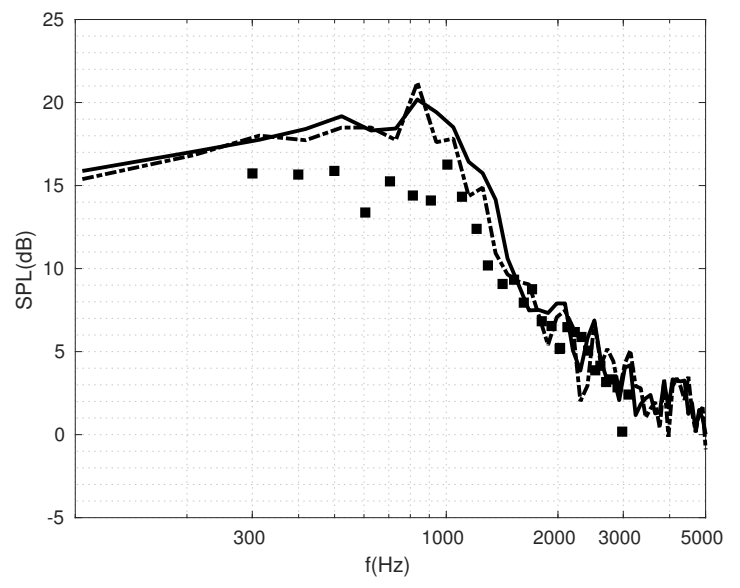

Fig. 7 Far-field SPL at an angle $\theta=90^{\circ}$ and a distance $R=2 \mathrm{~m}$ above the airfoil trailing-edge: Experiment (symbols), time domain propagation (solid line), frequency domain propagation (dash-dot line).

\section{E. Computational time and data storage}

\section{Computational cost}

In the time domain approach, the RPM and DG simulations are simultaneously performed using 24 cores on the Vlaams Supercomputer ThinKing. The computation lasts 1907 CPU hours. For the frequency domain approach, the RPM simulation is performed using 24 cores on the supercomputer ThinKing, whereas the FEM simulation is carried out using 1 core on a standard computer Intel Core i7 $2.7 \mathrm{GHz}, 32 \mathrm{~GB}$ RAM. In the latter case, the RPM source computation lasts $71.6 \mathrm{CPU}$ hours. The FEM simulation time is of about 5 hours for 100 frequencies, which is equivalent to a computational time per frequency of 3 minutes. As already mentioned above, the simulation cost is driven by the RPM computation. For the present airfoil case, the CPU cost is 30 times higher using the time domain approach, due to the small time step used in the DG code to respect the CFL restriction. This limitation may be alleviated by the use of implicit, or multi-time stepping schemes for the time integration.

\section{Data storage}

In the frequency domain computational strategy, the sound sources computed from RPM have to be first stored on disk before performing the FEM simulation. For the airfoil case presented above it corresponds approximately to $3.4 \mathrm{~Gb}$ of data.

\section{Conclusion}

In this study, a computational aeroacoustic strategy for low Mach number flow applications is presented. It combines the RPM method with a sound propagation model in the frequency domain. Discarding the mean flow convection effects and introducing time-harmonic assumption in the acoustic perturbation equations, the sound propagation model is equivalent to a non-homogeneous Helmholtz equation. The source term in the Helmholtz equation is computed from turbulent velocity fluctuations stochastically generated by the RPM method. The Helmholtz problem is then solved using a high-order finite element framework. This numerical strategy is applied in 2D to predict the trailing-edge noise radiated from a controlled-diffusion airfoil. The sound pressure level and directivities predicted in the far-field region are compared with the spectra calculated from a time-domain propagation solver. Comparisons are also performed with experimental data extracted from the literature. The results obtained from the time domain and the frequency domain solvers show a good agreement. An over-prediction of the sound levels by 2 to $5 \mathrm{~dB}$ is reported compared to the experimental data at low frequencies. 

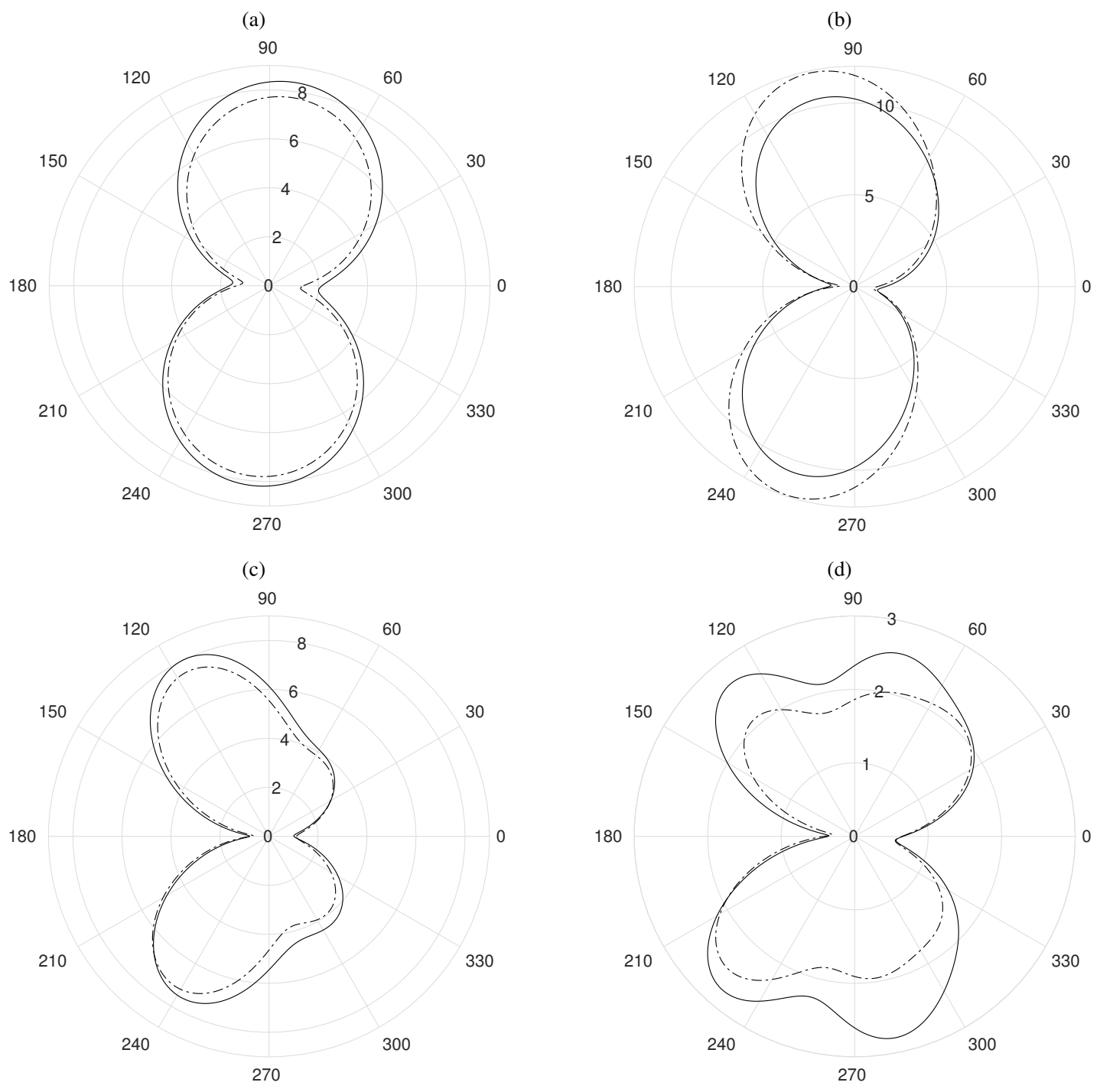

Fig. 8 Directivity plots of the pressure amplitude at a distance $R=2 \mathrm{~m}$ from the airfoil trailing-edge: time domain propagation (solid line), frequency domain propagation (dash-dot line) for frequencies of (a) $417 \mathrm{~Hz}$, $k_{c}=1.04$; (b) $835 \mathrm{~Hz}, k_{c}=2.09$; (c) $1253 \mathrm{~Hz}, k_{c}=3.14$ and (d) $1880 \mathrm{~Hz}, k_{c}=4.71$, where $k_{c}$ is the Helmholtz number based on the chord length. The pressure is normalized with respect to the reference pressure $p_{\text {ref }}$.

\section{Appendix}

\section{A. Integral length scale and integral time scale calculation}

The turbulence integral length scale $\Lambda$ and integral time scale $\tau$ are obtained from the turbulent kinetic energy $k$ and specific dissipation rate $\omega_{t}$ as follows:

$$
\begin{aligned}
\Lambda & =\frac{c_{l} \sqrt{k}}{C_{\mu} \omega_{t}}, \\
\tau & =\frac{2}{\beta C_{0} \omega_{t}},
\end{aligned}
$$

where $c_{l}=0.54, C_{\mu}=0.09, \beta=0.09$, and $C_{0}=2.1$ are the constants of the SST $k-\omega$ turbulence model. 


\section{B. Numerical sensitivity study of the source mapping schemes}

In this section, the numerical sensitivity of the acoustic results with respect to the source mapping scheme is discussed. For this purpose, the pressure spectra at a distance $R=2 \mathrm{~m}$ above the airfoil trailing-edge are computed using the three source mapping schemes presented in section II.E. The acoustic propagation is carried out in the frequency domain using source interpolation orders of $p_{\text {source }}=3$ and $p_{\text {source }}=10$. For the inverse distance weighting interpolation, a radius $R_{\mathrm{ac}}=10 h_{\mathrm{RPM}}$ is chosen.

(a)

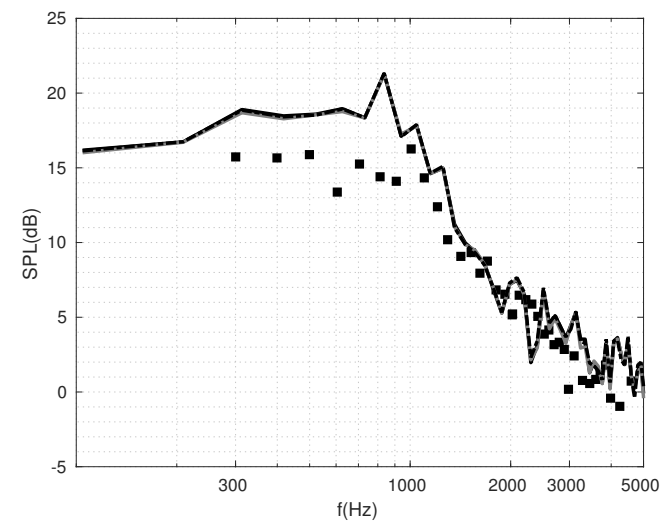

(b)

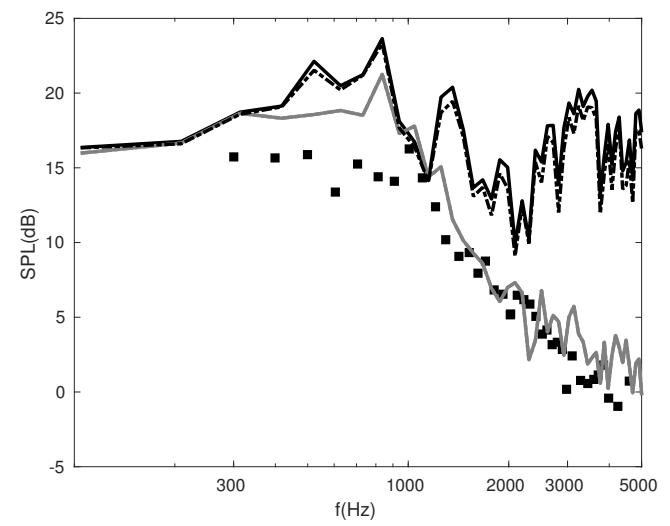

Fig. 9 Far-field SPL at an angle $\theta=90^{\circ}$ and a distance $R=2 \mathrm{~m}$ above the airfoil trailing-edge: experiment (symbols), spline interpolation (black-solid line), inverse distance weighting interpolation (gray-solid line), linear interpolation (black-dash-dot line), with source interpolation order (a) $p_{\text {source }}=10$ and (b) $p_{\text {source }}=3$.

Fig. 9(a) presents the far-field SPL obtained using the three mapping schemes for a source interpolation order $p_{\text {source }}=10$. The three spectra are in excellent agreement, with only slight differences at high frequencies. This indicates that for a high enough order $p_{\text {source}}$, the choice of the mapping strategy only has a marginal influence on the quality of the results.

The far-field SPL computed with the three mapping schemes for the order $p_{\text {source }}=3$ are displayed in Fig. 9(b). The spectra do not overlap in this case. While the inverse distance weighting interpolation provides results in good agreement with the reference numerical spectra (obtained using $p_{\text {source }}=10$ ), the linear and spline interpolation methods overpredict the sound pressure levels for frequencies $f>300 \mathrm{~Hz}$. This may be attributed to the fact that the linear and spline interpolation schemes have a small support, as determined by the fine RPM grid size, which in turn leads to an under sampling of the source field distribution, particularly at high frequencies. On the contrary, the inverse distance weighting approach, because it provides a larger support $R_{\mathrm{ac}}$, is more robust for lower-order source interpolation.

\section{Acknowledgments}

The authors acknowledge with thanks the support of the European Commission's Framework Program "Horizon 2020", through the Marie Skłodowska-Curie Innovative Training Networks (ITN) "AEOLUS4FUTURE - Efficient harvesting of the wind energy" (H2020-MSCA-ITN-2014: Grant agreement no. 643167) to the present research project.

"The computational resources and services used in this work were provided by the VSC (Flemish Supercomputer Center), funded by the Research Foundation - Flanders (FWO) and the Flemish Government - department EWI".

\section{References}

[1] Bailly, C., Bogey, C., and Marsden, O., "Progress in direct noise computation," International Journal of Aeroacoustics, Vol. 9 , No. 1-2, 2010, pp. 123-143.

[2] Ewert, R., "RPM - the fast Random Particle-Mesh method to realize unsteady turbulent sound sources and velocity fields for CAA applications," 13th AIAA/CEAS Aeroacoustics Conference, 2007, p. 3506. 
[3] Ewert, R., Appel, C., Dierke, J., and Herr, M., "RANS/CAA Based Prediction of NACA 0012 Broadband Trailing Edge Noise and Experimental Validation," 15th AIAA/CEAS Aeroacoustics Conference, 2009, p. 3269.

[4] Ewert, R., Dierke, J., Siebert, J., Neifeld, A., Appel, C., Siefert, M., and Kornow, O., "CAA broadband noise prediction for aeroacoustic design," Journal of Sound and Vibration, Vol. 330, No. 17, 2011, pp. 4139-4160.

[5] Ewert, R., and Schröder, W., "Acoustic perturbation equations based on flow decomposition via source filtering," Journal of Computational Physics, Vol. 188, No. 2, 2003, pp. 365-398.

[6] Rautmann, C., Dierke, J., Ewert, R., Hu, N., and Delfs, J., "Generic Airfoil Trailing-Edge Noise Prediction using Stochastic Sound Sources from Synthetic Turbulence,” 20th AIAA/CEAS Aeroacoustics Conference, 2014, p. 3298.

[7] Dieste, M., and Gabard, G., "Random Particle Methods Applied to Broadband Fan Interaction Noise," Journal of Computational Physics, Vol. 231, No. 24, 2012, pp. 8133-8151.

[8] Allan, M. R., and Darbyshire, O. R., "Comparison of LES and Stochastic Source Generation Methods for Aero- and Hydro-Acoustic Design Guidance,” 20th AIAA/CEAS Aeroacoustics Conference, 2014, p. 2319.

[9] Cozza, I. F., Iob, A., and Arina, R., "Broadband trailing-edge noise prediction with a stochastic source model," Computers and Fluids, Vol. 57, 2012, pp. 98-109.

[10] Casalino, D., and Barbarino, M., "Stochastic Method for Airfoil Self-Noise Computation in Frequency Domain," AIAA Journal, Vol. 49, No. 11, 2011, pp. 2453-2469.

[11] Howe, M. S., "Contributions to the theory of aerodynamic sound, with application to excess jet noise and the theory of the flute," Journal of Fluid Mechanics, Vol. 71, No. 04, 1975, pp. 625-673.

[12] Bériot, H., Prinn, A., and Gabard, G., "Efficient implementation of high-order finite elements for Helmholtz problems," International Journal for Numerical Methods in Engineering, Vol. 106, No. 3, 2016, pp. 213-240.

[13] Moreau, S., and Roger, M., "Effect of Airfoil Aerodynamic Loading on Trailing Edge Noise Sources," AIAA Journal, Vol. 43, No. 1, 2005, pp. 41-52.

[14] Pope, S. B., Turbulent flows, Cambridge University Press, 2000.

[15] Kadar, A., Martinez-Lera, P., Tournour, M., Schram, C. F., De Roeck, W., and Desmet, W., "Trailing-edge noise prediction using synthetic turbulence and acoustic perturbation equations in a hybrid methodology," 23rd AIAA/CEAS Aeroacoustics Conference, 2017, p. 3846.

[16] Toulorge, T., and Desmet, W., "Curved Boundary Treatments for the Discontinuous Galerkin Method Applied to Aeroacoustic Propagation,” AIAA Journal, Vol. 48, No. 2, 2010, pp. 479-489.

[17] Bermúdez, A., Hervella-Nieto, L., Prieto, A., Rodrı, R., et al., "An optimal perfectly matched layer with unbounded absorbing function for time-harmonic acoustic scattering problems," Journal of Computational Physics, Vol. 223, No. 2, 2007, pp. 469-488.

[18] Solin, P., Segeth, K., and Dolezel, I., Higher-Order Finite Element Methods, Chapman \& Hall/CRC, 2004.

[19] Toulorge, T., and Desmet, W., "CFL Conditions for Runge-Kutta discontinuous Galerkin methods on triangular grids," Journal of Computational Physics, Vol. 230, No. 12, 2011, pp. 4657-4678.

[20] Martínez-Lera, P., "Modeling of aerodynamic noise production and flow-acoustic feedback for wall-bounded low Mach number flows," Ph.D. thesis, KU Leuven, 2010.

[21] Moreau, S., Neal, D., and Foss, J., "Hot-Wire Measurements Around a Controlled Diffusion Airfoil in an Open-Jet Anechoic Wind Tunnel," Journal of Fluids Engineering, Vol. 128, No. 4, 2006, pp. 699-706.

[22] Wang, M., Moreau, S., Iaccarino, G., and Roger, M., "LES prediction of wall-pressure fluctuations and noise of a low-speed airfoil,” International Journal of Aeroacoustics, Vol. 8, No. 3, 2009, pp. 177-197.

[23] Christophe, J., “Application of Hybrid Methods to High Frequency Aeroacoustics,” Ph.D. thesis, Université libre de Bruxelles, 2011.

[24] Martínez-Lera, P., Christophe, J., and Schram, C., "Computation of the self-noise of a controlled-diffusion airfoil based on the acoustic analogy," International Journal of Aeroacoustics, Vol. 16, No. 1-2, 2017, pp. 44-64. 
[25] Weller, H. G., Tabor, G., Jasak, H., and Fureby, C., "A tensorial approach to computational continuum mechanics using object-oriented techniques," Computers in physics, Vol. 12, No. 6, 1998, pp. 620-631.

[26] Vanelderen, B., De Roeck, W., and Desmet, W., "Flow noise prediction of confined flows using synthetic turbulence and linearized Euler equations in a hybrid methodology," 19th AIAA/CEAS Aeroacoustics Conference, 2013, p. 2267.

[27] Korchagin, V., Vanelderen, B., Roeck, W. D., and Desmet, W., "Validation of the 3D stochastic turbulence reconstruction method with the Gaussian filter," International Conference on Noise and Vibration Engineering, ISMA-USD, Leuven, BE, 2016, pp. 363-372.

[28] Kadar, A. H., Martinez-Lera, P., Korchagin, V., Roeck, W. D., and Desmet, W., "Stochastic reconstruction of turbulence for trailing-edge noise computation," International Conference on Noise and Vibration Engineering, ISMA-USD, Leuven, BE, 2016, pp. 347-362.

[29] Toulorge, T., "Efficient Runge-Kutta Discontinuous Galerkin Methods Applied to Aeroacoustics," Ph.D. thesis, KU Leuven, 2012.

[30] Reymen, Y., “3D High-order Discontinuous Galerkin Methods for Time-Domain Simulation of Flow Noise Propagation,” Ph.D. thesis, KU Leuven, 2008.

[31] Scott, J., Pilon, A., Lyrintzis, A., and Rozmajzl, T., “A numerical investigation of noise from a rectangular jet," 35th Aerospace Sciences Meeting and Exhibit, 1997, p. 285. 